\title{
Transdermal estrogen prevents cyclically-recurrent genital herpes
}

\section{Peter Greenhouse FRCOG Bristol Sexual Health Centre, Bristol UK peter.greenhouse@nhs.net}

In the early classic descriptions of genital herpes by Unna (1883),

Diday \& Doyon (1886) and Berg (1889), over three quarters of

their cases in women presented immediately pre-menstrually, the

condition being called "Herpes menstrualis" or "catamenial herpes".

Very little has appeared in the English scientific literature since then on

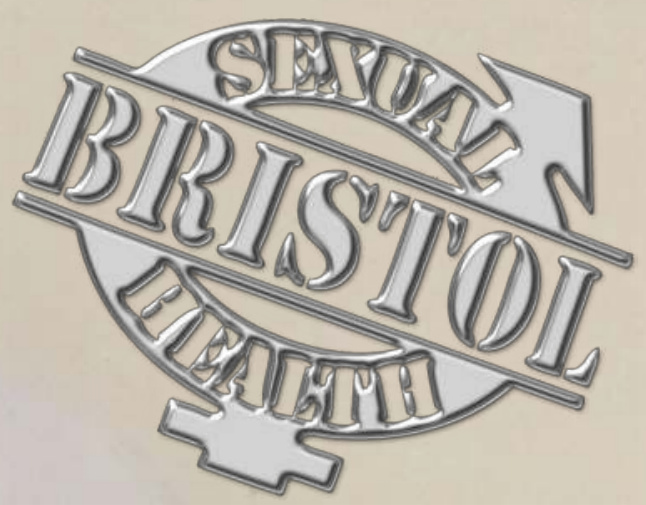

cyclically-recurrent genital herpes $(\mathrm{CH})$. Anecdotally, we have shown that

this can be effectively prevented by using aciclovir only in the luteal phase ${ }^{1}$

Applying the basic biologically plausible principle that:

"Any physical or mental symptom with a precise cyclical timing must have an hormonal cause, and therefore possibly, an hormonal solution" we observed that $\mathrm{CH}$ is more likely to happen in perimenopausal women whose immunity is diminished by severe premenstrual syndrome (sPMS), which itself is treatable with transdermal estradiol taken throughout the luteal phase, or continuously daily in women with a Levonorgestrel-IUS ${ }^{2}$

AIM: To observe the frequency of herpes recurrences in women commencing estradiol treatment for perimenopausal sPMS

METHODS: Proof of concept study reviewing 12 perimenopausal women median age 41 years (range 36-45) seen between 2005-15, each meeting the criteria for sPMS subsequently defined in DSM-5. Each had at least 4 consecutive monthly episodes of $\mathrm{CH}$ (culture-proven HSV2) before some took luteal-phase aciclovir, then all switched to transdermal 17-beta estradiol gel (Sandrena ${ }^{\circledR}$ ) $0.5 \mathrm{mg}$ daily for 14 days in the luteal phase or continuously daily for those using Mirena (LNG-IUS).

Subjects self-reported mood parameters and herpetic symptoms with a modified daily symptom chart over nine months follow-up during which no aciclovir was taken pre-emptively, but was available in case of episodic recurrence.

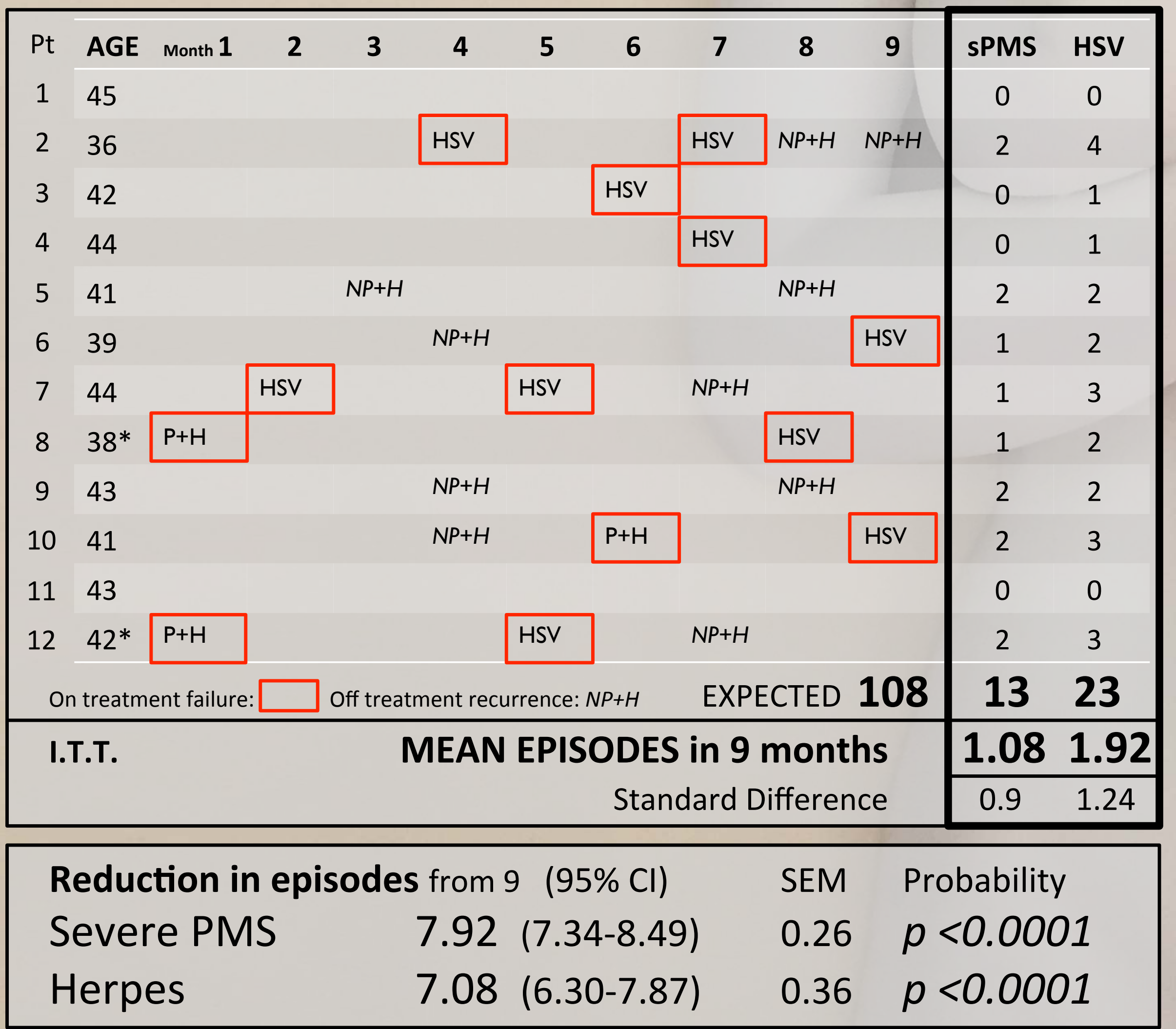

ANALYSIS:

Intention-to-treat analysis using Student's t-test included 10 cycles where estradiol treatment was omitted in error and both conditions recurred in each untreated cycle.

$(N P+H$ on table)

\section{RESULTS: - see table}

All women experienced substantial or complete relief of SPMS in all but three cycles when treatment was correctly taken.

Two women* in whom mood symptoms were not initially controlled and herpes recurred in the first month, responded successfully when the estradiol dose was increased to $1 \mathrm{mg} / \mathrm{d}$.

Only 13 symptomatic herpes episodes occurred when estradiol treatment was correctly taken in 108 woman-months of observation.

\section{CONCLUSIONS:}

Transdermal estradiol treatment is a novel and biologically plausible method of preventing cyclically-recurrent genital herpes. It stabilises the hormonal milieu suppressing cyclical fluctuations which would normally downregulate T-helper 1 cells in the luteal phase - this immune suppression is exacerbated in the perimenopause and by PMS.

Most women with severe perimenopausal premenstrual syndrome are seen by GPs who prescribe ineffective SSRI drugs. Most women with catamenial herpes are seen in sexual health clinics and offered only suppressive or episodic aciclovir. This study demonstrates that both conditions can be managed simultaneously with an integrated approach to women's health.

1. Greenhouse P, Evans AE. Premenstrually-recurrent genital herpes in perimenopausal women: Prevention by luteal phase aciclovir. ISSTDR Amsterdam 2005

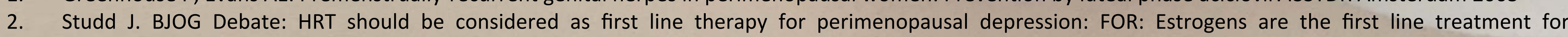
perimenopausal women. BJOG 2016: 123; 1011 Graphic Design: PG Sculpture: “Modesty” (C2008 by Jamie McCartney 